\title{
Bloch's theory in periodic structures with Rashba's spin-orbit interaction
}

\author{
S. Smirnov ${ }^{1}$, D. Bercioux ${ }^{1,2}$ and M. Grifoni ${ }^{1}$ \\ 1 Institut für Theoretische Physik, Universität Regensburg, D-93040 Regensburg, Germany \\ 2 Physikalische Institut, Albert-Ludwigs-Universität, D-79104 Freiburg, Germany
}

PACS 72.25.Dc - Spin polarized transport in semiconductors

PACS 73.21. Hb - Quantum wires

PACS 73.23.-b - Electronic transport in mesoscopic systems

\begin{abstract}
We consider a two-dimensional electron gas with Rashba's spin-orbit interaction and two in-plane potentials superimposed along directions perpendicular to each other. The first of these potentials is assumed to be a general periodic potential while the second one is totally arbitrary. A general form for Bloch's amplitude is found and an eigen-value problem for the band structure of the system is derived. We apply the general result to the two particular cases in which either the second potential represents a harmonic in-plane confinement or it is zero. We find that for a harmonic confinement regions of the Brillouin zone with high polarizations are associated with the ones of large group velocity.
\end{abstract}

Introduction. - It is well known that in a two' dimensional electron gas (2DEG) formed in a semiconductor by an asymmetric confining potential Rashba's spinorbit interaction (RSOI) [1] plays an important role. It is very attractive for applications in electronic devices because the spin-orbit coupling strength can be controlled by , an external gate voltage [2]. Other spin-orbit mechanisms ' such as Dresselhaus' spin-orbit interaction [3] can be relevant. In this work, for simplicity, we focus on the effects of RSOI since Dresselhaus' term can be treated in full analogy. Additionally, when externally enhanced, RSOI may become stronger than other spin-orbit interactions. In this case the Hamiltonian has the form:

$$
\hat{H}_{0}^{2 \mathrm{D}}=\frac{\hbar^{2} \hat{\mathbf{k}}^{2}}{2 m}-\frac{\hbar^{2} k_{\mathrm{so}}}{m}\left(\hat{\sigma}_{x} \hat{k}_{z}-\hat{\sigma}_{z} \hat{k}_{x}\right),
$$

where $k_{\text {so }}$ is the spin-orbit coupling strength and the operator $\hat{\mathbf{k}}$ is related to the momentum operator $\hat{\mathbf{p}}$ as $\hat{\mathbf{p}}=\hbar \hat{\mathbf{k}}$. The eigen-states of (1) have a two-dimensional spinorial part $\phi_{\lambda}^{2 \mathrm{D}}(\sigma)$ where $\lambda=1,2$ is the eigen-state index called chirality and $\sigma= \pm 1$ is the spin index. The eigen-energies split into two branches $\varepsilon_{\lambda}^{2 \mathrm{D}}\left(k_{x}, k_{z}\right)$. Systems with such energy spectrum can be exploited to study spin-dependent transport in different semiconductors, especially in III-V compounds because of the large values of the spin-orbit coupling strength. As mentioned above they are also used to build both two-dimensional (2D) and essentially one-dimensional (1D) electronic devices. One such device, called spin transistor, was proposed in Ref. [4] for the case of a quasi-1D system with RSOI. It is obtained from the 2DEG described by (1), where by further confinement along, e.g. the $z$-direction, a quasi-1D wire is formed. These quasi-1D systems were investigated for the case of a harmonic $z$-confinement $[5,6]$ and for an infinite square well $z$-confinement [7]. In general one can conceive a situation where an arbitrary potential $V(z)$ along the $z$-direction is present. We would like to emphasize that $V(z)$ must not necessarily be a confinement. In this case the Hamiltonian is written as:

$$
\hat{H}_{z}^{2 \mathrm{D}}=\frac{\hbar^{2} \hat{\mathbf{k}}^{2}}{2 m}+V(\hat{z})-\frac{\hbar^{2} k_{\mathrm{so}}}{m}\left(\hat{\sigma}_{x} \hat{k}_{z}-\hat{\sigma}_{z} \hat{k}_{x}\right) .
$$

The Hamiltonian $\hat{H}_{z}^{2 \mathrm{D}}$ assumes that the spin-orbit interaction caused by $V(z)$ is much weaker than RSOI induced by an asymmetric confinement forming the 2DEG. For a given system this means that the out-of-plane electric field should be much stronger than the in-plane one.

In systems described by (2) RSOI removes the spin degeneracy of each energy branch and splits them into two ones. The splitting is also accompanied by a deviation from the quadratic dependence on the momentum. For example in the case of a harmonic $z$-confinement, if only the first two transverse sub-bands are considered, there are four $1 \mathrm{D}$ dispersion relations $\varepsilon_{\chi}^{1 \mathrm{D}}\left(k_{x}\right), \chi=1,2,3,4$. 
The eigen-states have a four-dimensional spinorial part $\phi_{k_{x}, \chi}^{1 \mathrm{D}}(\sigma, j)$ where $j=0,1$ is the transverse mode index. It turns out that for this model the energy spectrum can be found in analytic form [6] from the diagonalization of Hamiltonian (2).

Structures where a periodic modulation $U(x)$ is additionally present have recently been investigated by various authors. For the case $U(x) \neq 0, V(z)=0$ the Bloch band energies have been found in Ref. [8] within the tightbinding approximation. In Ref. [9] the same problem has been investigated numerically. In the presence of an external homogeneous magnetic field the so-called magnetic Bloch states are discussed in Ref. [10] for the case $U(x) \neq 0$ and $V(z)$ being periodic as well. However, analytic relations between the eigen-values of those problems and the ones of their corresponding truly 1D problems without RSOI have not been provided so far and this is one of the topics of the present letter.

In this work we consider two potentials $U(x)$ and $V(z)$, where the potential $U(x)$ is a periodic potential while the shape of the potential $V(z)$ is arbitrary. First, a general structure of the Bloch amplitude is educed. Next, we formulate the eigen-value problem. As an example we apply the general approach to the particular case of a harmonic confinement and as a consequence generalize the analytical results obtained in Ref. [6] to the case of a periodic potential along the wire. Finally, setting $V(z)=0$ we analytically and exactly solve the problem examined numerically in [9] and find qualitative differences from some of the numerical results obtained in [9].

A periodic structure with RSOI. - In this section we consider a system described by the Hamiltonian $\hat{H}_{z}^{2 \mathrm{D}}+$ $U(\hat{x})$ where the $1 \mathrm{D}$ periodic potential $U(x)$ has the period $L$ :

$$
U(x+L)=U(x) .
$$

That is, the total Hamiltonian of the problem is

$$
\hat{H}=\frac{\hbar^{2} \hat{\mathbf{k}}^{2}}{2 m}+V(\hat{z})-\frac{\hbar^{2} k_{\mathrm{so}}}{m}\left(\hat{\sigma}_{x} \hat{k}_{z}-\hat{\sigma}_{z} \hat{k}_{x}\right)+U(\hat{x}) .
$$

Before considering the full problem it is instructive to refresh the Bloch theorem for a truly 1D periodic structure without RSOI.

A truly $1 D$ periodic structure. As it is known [11], a system described by the Hamiltonian

$$
\hat{H}_{0}^{1 \mathrm{D}}=\frac{\hbar^{2} \hat{k}_{x}^{2}}{2 m}+U(\hat{x})
$$

has eigen-energies $\varepsilon_{l, \sigma}^{(0)}\left(k_{\mathrm{B}}\right)$ and eigen-states $\left|l, k_{\mathrm{B}}, \sigma\right\rangle$, with

$$
\hat{H}_{0}^{1 \mathrm{D}}\left|l, k_{\mathrm{B}}, \sigma\right\rangle=\varepsilon_{l, \sigma}^{(0)}\left(k_{\mathrm{B}}\right)\left|l, k_{\mathrm{B}}, \sigma\right\rangle,
$$

characterized by Bloch's quasi-momentum $k_{\mathrm{B}}$, running over a discrete set of values in the first Brillouin zone, and the band index $l$. The eigen-energies are degenerate with respect to the spin index, $\varepsilon_{l,+1}^{(0)}\left(k_{\mathrm{B}}\right)=\varepsilon_{l,-1}^{(0)}\left(k_{\mathrm{B}}\right) \equiv$
$\varepsilon_{l}^{(0)}\left(k_{\mathrm{B}}\right)$. In the coordinate representation the eigen-state is related to Bloch's amplitude $u_{l, k_{\mathrm{B}}, \sigma}\left(x, \sigma^{\prime}\right)$ by

$$
\begin{aligned}
& \left\langle x, \sigma^{\prime} \mid l, k_{\mathrm{B}}, \sigma\right\rangle=\frac{1}{\sqrt{L_{0}}} e^{\mathrm{i} k_{\mathrm{B}} x} u_{l, k_{\mathrm{B}}, \sigma}\left(x, \sigma^{\prime}\right), \\
& u_{l, k_{\mathrm{B}}, \sigma}\left(x, \sigma^{\prime}\right)=\delta_{\sigma^{\prime}, \sigma} u_{l, k_{\mathrm{B}}}(x), \\
& u_{l, k_{\mathrm{B}}}(x)=u_{l, k_{\mathrm{B}}}(x+L),
\end{aligned}
$$

where $L_{0}$ is the size of the system along the $x$-axis. Here the spinorial structure of the Bloch amplitude is trivial.

Influence of a transverse potential and RSOI. The transverse potential $V(z)$ together with RSOI change the Bloch spinors $\left|l, k_{\mathrm{B}}, \sigma\right\rangle$. We denote the new spinors through $\left|l, k_{\mathrm{B}}, \eta\right\rangle$ :

$$
\hat{H}\left|l, k_{\mathrm{B}}, \eta\right\rangle=\varepsilon_{l, \eta}\left(k_{\mathrm{B}}\right)\left|l, k_{\mathrm{B}}, \eta\right\rangle .
$$

As a result the Bloch amplitude acquires a new spinorial structure:

$$
\begin{aligned}
& \left\langle x, j, \sigma \mid l, k_{\mathrm{B}}, \eta\right\rangle=\frac{1}{\sqrt{L_{0}}} e^{\mathrm{i} k_{\mathrm{B}} x} u_{l, k_{\mathrm{B}}, \eta}(x ; j, \sigma), \\
& u_{l, k_{\mathrm{B}}, \eta}(x ; j, \sigma)=u_{l, k_{\mathrm{B}}, \eta}(x+L ; j, \sigma),
\end{aligned}
$$

where $|j\rangle$ is an eigen-vector corresponding to an eigenvalue $\varepsilon_{j}^{z}$ and both are found from the Schrödinger equation:

$$
\left[\frac{\hbar^{2} \hat{k}_{z}^{2}}{2 m}+V(\hat{z})\right]|j\rangle=\varepsilon_{j}^{z}|j\rangle
$$

It is convenient to represent the total Hamiltonian (4) as the sum $\hat{H}=\hat{H}^{\prime}+\hat{H}^{\prime \prime}$, where $\hat{H}^{\prime}$ and $\hat{H}^{\prime \prime}$ are given by

$$
\begin{aligned}
& \hat{H}^{\prime} \equiv \frac{\hbar^{2}}{2 m}\left(\hat{k}_{x}+\hat{\sigma}_{z} k_{\mathrm{so}}\right)^{2}+U(\hat{x})+ \\
& +\frac{\hbar^{2} \hat{k}_{z}^{2}}{2 m}+V(\hat{z})-\frac{\hbar^{2} k_{\mathrm{so}}^{2}}{2 m}, \\
& \hat{H}^{\prime \prime} \equiv-\frac{\hbar^{2} k_{\mathrm{so}}}{m} \hat{\sigma}_{x} \hat{k}_{z} .
\end{aligned}
$$

The eigen-energies and eigen-states of $\hat{H}^{\prime}$ are easily found and related to $\varepsilon_{l}^{(0)}\left(k_{\mathrm{B}}\right)$ and $u_{l, k_{\mathrm{B}}}(x)$ as follows:

$$
\begin{aligned}
& \hat{H}^{\prime}\left|l, k_{\mathrm{B}}, j, \sigma\right\rangle=\varepsilon_{l, j, \sigma}^{\prime}\left(k_{\mathrm{B}}\right)\left|l, k_{\mathrm{B}}, j, \sigma\right\rangle, \\
& \varepsilon_{l, j, \sigma}^{\prime}\left(k_{\mathrm{B}}\right)=\varepsilon_{l}^{(0)}\left(k_{\mathrm{B}}+\sigma k_{\mathrm{So}}\right)-\frac{\hbar^{2} k_{\mathrm{So}}^{2}}{2 m}+\varepsilon_{j}^{z}, \\
& \left\langle x, j^{\prime}, \sigma^{\prime} \mid l, k_{\mathrm{B}}, j, \sigma\right\rangle=\frac{\delta_{j^{\prime}, j} \delta_{\sigma^{\prime}, \sigma} e^{\mathrm{i} k_{\mathrm{B}} x}}{\sqrt{L}_{0}} u_{l, k_{\mathrm{B}}+\sigma k_{\mathrm{so}}}(x) .
\end{aligned}
$$

Let us denote through $\theta_{l, k_{\mathrm{B}}, \eta}(j, \sigma)$ the Bloch spinors in the $\left\{l, k_{\mathrm{B}}, j, \sigma\right\}$ representation, that is $\theta_{l, k_{\mathrm{B}}, \eta}(j, \sigma) \equiv$ $\left\langle l, k_{\mathrm{B}}, j, \sigma \mid l, k_{\mathrm{B}}, \eta\right\rangle$. Then

$$
\left\langle l^{\prime}, k_{\mathrm{B}}^{\prime}, j, \sigma \mid l, k_{\mathrm{B}}, \eta\right\rangle=\delta_{l^{\prime}, l} \delta_{k_{\mathrm{B}}^{\prime}, k_{\mathrm{B}}} \theta_{l, k_{\mathrm{B}}, \eta}(j, \sigma) .
$$

We can make a general statement concerning Bloch's amplitude $u_{l, k_{\mathrm{B}}, \eta}(x ; j, \sigma)$. From the identity

$$
\begin{aligned}
& \frac{1}{\sqrt{L_{0}}} e^{\mathrm{i} k_{\mathrm{B}} x} u_{l, k_{\mathrm{B}}, \eta}(x ; j, \sigma) \equiv \\
& \equiv \sum_{l^{\prime}, k_{\mathrm{B}}^{\prime}, j^{\prime}, \sigma^{\prime}}\left\langle x, j, \sigma \mid l^{\prime}, k_{\mathrm{B}}^{\prime}, j^{\prime}, \sigma^{\prime}\right\rangle\left\langle l^{\prime}, k_{\mathrm{B}}^{\prime}, j^{\prime}, \sigma^{\prime} \mid l, k_{\mathrm{B}}, \eta\right\rangle
\end{aligned}
$$


and using (12) and (13) it follows

$$
u_{l, k_{\mathrm{B}}, \eta}(x ; j, \sigma)=u_{l, k_{\mathrm{B}}+\sigma k_{\mathrm{so}}}(x) \theta_{l, k_{\mathrm{B}}, \eta}(j, \sigma) .
$$

The last equation clearly shows that the spinorial part of the Bloch amplitude $u_{l, k_{\mathrm{B}}, \sigma}\left(x, \sigma^{\prime}\right)$ in eq. (7) transforms from $\delta_{\sigma^{\prime}, \sigma}$ into the spinor $\theta_{l, k_{\mathrm{B}}, \eta}(j, \sigma)$ when the potential $V(z)$ and RSOI are involved.

The spinors $\theta_{l, k_{\mathrm{B}}, \eta}(j, \sigma)$ can be found from eq. (8) which in $\left\{l, k_{\mathrm{B}}, j, \sigma\right\}$ representation takes the form:

$$
\begin{aligned}
& \sum_{j^{\prime}, \sigma^{\prime}}\left[\left\langle l^{\prime}, k_{\mathrm{B}}^{\prime}, j, \sigma\left|\hat{H}^{\prime}\right| l, k_{\mathrm{B}}, j^{\prime}, \sigma^{\prime}\right\rangle+\right. \\
& \left.+\left\langle l^{\prime}, k_{\mathrm{B}}^{\prime}, j, \sigma\left|\hat{H}^{\prime \prime}\right| l, k_{\mathrm{B}}, j^{\prime}, \sigma^{\prime}\right\rangle\right] \theta_{l, k_{\mathrm{B}}, \eta}\left(j^{\prime}, \sigma^{\prime}\right)= \\
& =\varepsilon_{l, \eta}\left(k_{\mathrm{B}}\right) \delta_{l^{\prime}, l} \delta_{k_{\mathrm{B}}^{\prime}, k_{\mathrm{B}}} \theta_{l, k_{\mathrm{B}}, \eta}(j, \sigma) .
\end{aligned}
$$

The matrix elements of $\hat{H}^{\prime}$ and $\hat{H}^{\prime \prime}$ are given by the expressions:

$$
\begin{aligned}
& \left\langle l^{\prime}, k_{\mathrm{B}}^{\prime}, j, \sigma\left|\hat{H}^{\prime}\right| l, k_{\mathrm{B}}, j^{\prime}, \sigma^{\prime}\right\rangle= \\
& =\varepsilon_{l, j, \sigma}^{\prime}\left(k_{\mathrm{B}}\right) \delta_{l^{\prime}, l} \delta_{k_{\mathrm{B}}^{\prime}, k_{\mathrm{B}}} \delta_{j, j^{\prime}} \delta_{\sigma, \sigma^{\prime}}, \\
& \left\langle l^{\prime}, k_{\mathrm{B}}^{\prime}, j, \sigma\left|\hat{H}^{\prime \prime}\right| l, k_{\mathrm{B}}, j^{\prime}, \sigma^{\prime}\right\rangle= \\
& =-\frac{\hbar^{2} k_{\mathrm{So}}}{m}\left\langle\sigma\left|\hat{\sigma}_{x}\right| \sigma^{\prime}\right\rangle\left\langle j\left|\hat{k}_{z}\right| j^{\prime}\right\rangle \delta_{l^{\prime}, l} \delta_{k_{\mathrm{B}}^{\prime}, k_{\mathrm{B}}},
\end{aligned}
$$

where $\left\langle\sigma\left|\hat{\sigma}_{x}\right| \sigma^{\prime}\right\rangle=1-\delta_{\sigma, \sigma^{\prime}}$. The final equation for the eigen-energies $\varepsilon_{l, \eta}\left(k_{\mathrm{B}}\right)$ and eigen-spinors $\theta_{l, k_{\mathrm{B}}, \eta}(j, \sigma)$ is obtained using eq. (16) together with eq. (17) by equating the band indices $l^{\prime}=l$ and Bloch's quasi-momenta $k_{\mathrm{B}}^{\prime}=k_{\mathrm{B}}$ :

$$
\begin{aligned}
& \sum_{j^{\prime}, \sigma^{\prime}}\left\{\delta_{j, j^{\prime}} \delta_{\sigma, \sigma^{\prime}}\left[\varepsilon_{l}^{(0)}\left(k_{\mathrm{B}}+\sigma k_{\mathrm{SO}}\right)+\varepsilon_{j}^{z}-\frac{\hbar^{2} k_{\mathrm{so}}^{2}}{2 m}\right]-\right. \\
& \left.-\frac{\hbar^{2} k_{\mathrm{So}}}{m}\left(1-\delta_{\sigma, \sigma^{\prime}}\right)\left\langle j\left|\hat{k}_{z}\right| j^{\prime}\right\rangle\right\} \theta_{l, k_{\mathrm{B}}, \eta}\left(j^{\prime}, \sigma^{\prime}\right)= \\
& =\varepsilon_{l, \eta}\left(k_{\mathrm{B}}\right) \theta_{l, k_{\mathrm{B}}, \eta}(j, \sigma) .
\end{aligned}
$$

We want to emphasize that eq. (18) can be applied to calculate the band structure for an arbitrary potential $V(z)$ and periodic potential $U(x)$ of a general form. Note the specific influence of the spin-orbit coupling: a) $j$-states are mixed by RSOI; b) the Bloch bands of the corresponding truly 1D problem with different $l$ are split into sub-bands independently, that is the splitting of band $l$ does not depend on the splitting of bands with $l^{\prime} \neq l$. Therefore as soon as the truly 1D band structure has been obtained, one can take any of its Bloch bands, let us say $l$, apply (18) to it and find the Bloch bands labeled with index $l$ in the presence of $V(z)$ and RSOI. The same inference remains valid if Dresselhaus' spin-orbit interaction is additionally included into the model.

Harmonic confinement. - Here we consider a particular case where the operator $V(\hat{z})$ represents a harmonic confinement of strength $\omega_{0}$. In this case the matrix elements of $\hat{k}_{z}$ are

$$
\left\langle j\left|\hat{k}_{z}\right| j^{\prime}\right\rangle= \pm \mathrm{i} \delta_{j, j^{\prime} \pm 1} \sqrt{\frac{\left(j+\frac{1}{2} \mp \frac{1}{2}\right) m \omega_{0}}{2 \hbar}} .
$$

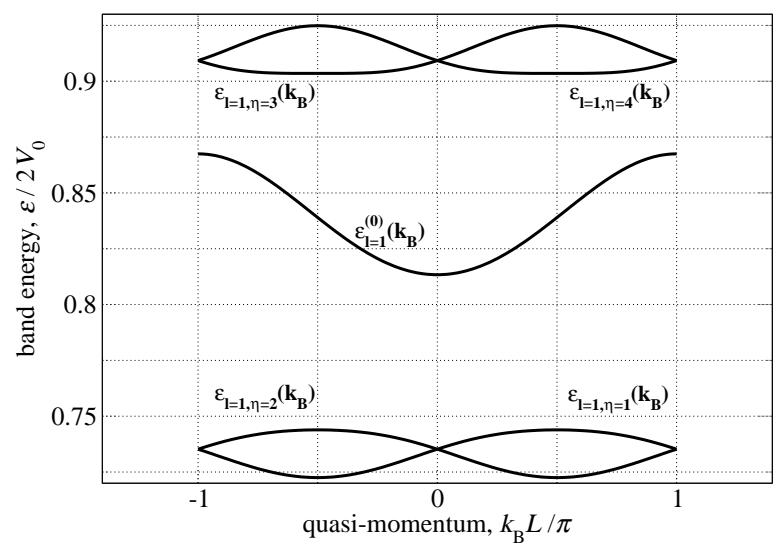

Fig. 1: The first Bloch band of the corresponding truly 1D system $\varepsilon_{l=1}^{(0)}\left(k_{\mathrm{B}}\right)$ together with the four Bloch sub-bands $\varepsilon_{l=1, \eta}\left(k_{\mathrm{B}}\right)$ of the quasi-1D system in the presence of RSOI and the transverse confinement.

If in (18) one keeps only the first two transverse modes, the problem reduces to the diagonalization of a $4 \times 4$ matrix and becomes solvable analytically. The validity of this approximation is discussed in Ref. [6]. After the diagonalization of (18), where now $j=0,1$, we obtain the following eigen-energies:

$$
\begin{aligned}
& \varepsilon_{l, \eta=1,2}\left(k_{\mathrm{B}}\right)=\varepsilon_{l}^{+}\left(k_{\mathrm{B}}\right)-\Xi_{l_{1,2}}\left(k_{\mathrm{B}}\right), \\
& \varepsilon_{l, \eta=3,4}\left(k_{\mathrm{B}}\right)=\varepsilon_{l}^{+}\left(k_{\mathrm{B}}\right)+\Xi_{l_{2,1}}\left(k_{\mathrm{B}}\right),
\end{aligned}
$$

where

$$
\begin{aligned}
& \varepsilon_{l}^{+}\left(k_{\mathrm{B}}\right) \equiv \frac{\varepsilon_{l}^{(0)}\left(k_{\mathrm{B}}+k_{\mathrm{so}}\right)+\varepsilon_{l}^{(0)}\left(k_{\mathrm{B}}-k_{\mathrm{so}}\right)}{2}+ \\
& +\hbar \omega_{0}-\frac{\hbar^{2} k_{\mathrm{so}}^{2}}{2 m}, \\
& \Xi_{l_{1,2}}\left(k_{\mathrm{B}}\right) \equiv \sqrt{\Xi^{2}+\left(\varepsilon_{l}^{-}\left(k_{\mathrm{B}}\right) \mp \frac{\hbar \omega_{0}}{2}\right)^{2}}, \\
& \varepsilon_{l}^{-}\left(k_{\mathrm{B}}\right) \equiv \frac{\varepsilon_{l}^{(0)}\left(k_{\mathrm{B}}+k_{\mathrm{so}}\right)-\varepsilon_{l}^{(0)}\left(k_{\mathrm{B}}-k_{\mathrm{so}}\right)}{2} \\
& \Xi \equiv \frac{\hbar^{2} k_{\mathrm{so}}}{m} \sqrt{\frac{m \omega_{0}}{2 \hbar}} .
\end{aligned}
$$

Since $\varepsilon_{l}^{(0)}\left(k_{\mathrm{B}}\right)=\varepsilon_{l}^{(0)}\left(-k_{\mathrm{B}}\right)$, the relations between the eigen-energies (20) follow:

$$
\begin{aligned}
& \varepsilon_{l, \eta=1}\left(k_{\mathrm{B}}\right)=\varepsilon_{l, \eta=2}\left(-k_{\mathrm{B}}\right), \\
& \varepsilon_{l, \eta=3}\left(k_{\mathrm{B}}\right)=\varepsilon_{l, \eta=4}\left(-k_{\mathrm{B}}\right),
\end{aligned}
$$

as expected due to the existence of both the time reversal symmetry and band overlap [12]. In fig. 1 we show the first Bloch band of the corresponding truly 1D problem and the four Bloch sub-bands growing out of it under the influence of RSOI and the transverse confinement. The spin-orbit coupling strength is chosen such that $L k_{\text {so }}=\pi / 2$. The periodic potential has the form:

$$
U(x)=V_{0}\left[1-\cos \left(\frac{2 \pi}{L} x\right)\right] .
$$




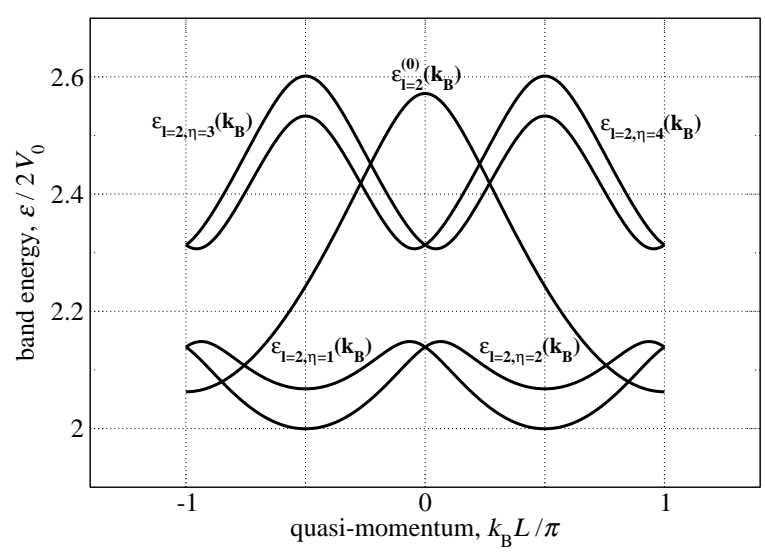

Fig. 2: The second Bloch band of the corresponding truly 1D system $\varepsilon_{l=2}^{(0)}\left(k_{\mathrm{B}}\right)$ together with the four Bloch sub-bands $\varepsilon_{l=2, \eta}\left(k_{\mathrm{B}}\right)$ of the quasi-1D system in the presence of RSOI and the transverse confinement.

The second Bloch band and its four sub-bands are plotted in fig. 2. It can be seen that for $l=1$ the Bloch band of the truly 1D problem without RSOI and its four sub-bands for the quasi-1D system with RSOI are all under the potential barrier while for $l=2$ they are above it. As usual RSOI does not remove the spin degeneracy at $k_{\mathrm{B}}=0$. It follows from (20) that the bands split when $\varepsilon_{l}^{-}\left(k_{\mathrm{B}}\right) \neq 0$. The derivative of the function $\varepsilon_{l}^{-}\left(k_{\mathrm{B}}\right)$ at $k_{\mathrm{B}}=0$ is easily found from (21):

$$
\left.\frac{d \varepsilon_{l}^{-}\left(k_{\mathrm{B}}\right)}{d k_{\mathrm{B}}}\right|_{k_{\mathrm{B}}=0}=v_{l}^{(0)}\left(k_{\mathrm{so}}\right),
$$

where $v_{l}^{(0)}\left(k_{\mathrm{B}}\right)$ is the group velocity of the corresponding truly 1D problem. Since for the chosen parameters the group velocity in (24) is not equal to zero (see figs. 1 and 21), it follows from (20) and (21) that the band splitting near the point $k_{\mathrm{B}}=0$ is linear in $k_{\mathrm{B}}$. This is also the case for a 2DEG where the linear momentum-dependence of the splitting is observed experimentally [13].

The corresponding normalized eigen-spinors $\theta_{l, k_{\mathrm{B}}, \eta}(j, \sigma)$ are expressed in terms of non-normalized ones, denoted through $\tilde{\theta}_{l, k_{\mathrm{B}}, \eta}(j, \sigma)$, as:

$$
\begin{aligned}
& \theta_{l, k_{\mathrm{B}}, \eta=1,4}=N_{l, k_{\mathrm{B}}, \eta=1,4}^{-\frac{1}{2}} \tilde{\theta}_{l, k_{\mathrm{B}}, \eta=1,4}, \\
& \theta_{l, k_{\mathrm{B}}, \eta=2,3}=N_{l, k_{\mathrm{B}}, \eta=2,3}^{-\frac{1}{2}} \tilde{\theta}_{l, k_{\mathrm{B}}, \eta=2,3},
\end{aligned}
$$

where

$$
\begin{aligned}
& \tilde{\theta}_{l, k_{\mathrm{B}}, \eta=1,4} \equiv\left[\begin{array}{c}
\frac{\mathrm{i}}{\Xi}\left[\varepsilon_{l}^{-}\left(k_{\mathrm{B}}\right)-\frac{\hbar \omega_{0}}{2} \mp \Xi_{l_{1}}\left(k_{\mathrm{B}}\right)\right] \\
0 \\
0 \\
1
\end{array}\right], \\
& \tilde{\theta}_{l, k_{\mathrm{B}}, \eta=2,3} \equiv\left[\begin{array}{c}
0 \\
-\frac{\mathrm{i}}{\Xi}\left[\varepsilon_{l}^{-}\left(k_{\mathrm{B}}\right)+\frac{\hbar \omega_{0}}{2} \pm \Xi_{l_{2}}\left(k_{\mathrm{B}}\right)\right] \\
1 \\
0
\end{array}\right] .
\end{aligned}
$$

We have introduced the notation

$$
\theta_{l, k_{\mathrm{B}}, \eta} \equiv\left[\begin{array}{l}
\theta_{l, k_{\mathrm{B}}, \eta}(j=0, \sigma=+1) \\
\theta_{l, k_{\mathrm{B}}, \eta}(j=0, \sigma=-1) \\
\theta_{l, k_{\mathrm{B}}, \eta}(j=1, \sigma=+1) \\
\theta_{l, k_{\mathrm{B}}, \eta}(j=1, \sigma=-1)
\end{array}\right],
$$

and an analogous one for the non-normalized spinor $\tilde{\theta}_{l, k_{\mathrm{B}}, \eta}$. In (25) $N_{l, k_{\mathrm{B}}, \eta}$ are the normalization constants:

$$
N_{l, k_{\mathrm{B}}, \eta}=\sum_{j=0}^{1} \sum_{\sigma=-1}^{+1}\left|\tilde{\theta}_{l, k_{\mathrm{B}}, \eta}(j, \sigma)\right|^{2} .
$$

Note that using (21) and (25) one gets the relations

$$
N_{l, k_{\mathrm{B}}, \eta=1,4}=N_{l,-k_{\mathrm{B}}, \eta=2,3} .
$$

For $k_{\text {so }} \rightarrow 0$ the spinors in (25) take the form:

$$
\theta_{l, k_{\mathrm{B}}, \eta=1,2,3,4}=\left[\begin{array}{c}
-\mathrm{i} \\
0 \\
0 \\
0
\end{array}\right],\left[\begin{array}{c}
0 \\
-\mathrm{i} \\
0 \\
0
\end{array}\right],\left[\begin{array}{l}
0 \\
0 \\
1 \\
0
\end{array}\right],\left[\begin{array}{l}
0 \\
0 \\
0 \\
1
\end{array}\right] .
$$

In the limit $U(x) \rightarrow 0$ we have $\varepsilon_{l}^{(0)}\left(k_{\mathrm{B}}\right) \rightarrow \hbar^{2} k_{B}^{2} / 2 m$, $u_{l, k_{\mathrm{B}}}(x) \rightarrow 1$ and from (20) and (21) we get:

$$
\begin{aligned}
& \varepsilon_{l, \eta=1,2}\left(k_{x}\right) \rightarrow \frac{\hbar^{2} k_{x}^{2}}{2 m}+\hbar \omega_{0}-\Xi_{1,2}^{(0)}\left(k_{x}\right), \\
& \varepsilon_{l, \eta=3,4}\left(k_{x}\right) \rightarrow \frac{\hbar^{2} k_{x}^{2}}{2 m}+\hbar \omega_{0}+\Xi_{2,1}^{(0)}\left(k_{x}\right),
\end{aligned}
$$

where

$$
\Xi_{1,2}^{(0)}\left(k_{x}\right) \equiv \sqrt{\Xi^{2}+\left(\frac{\hbar^{2} k_{x} k_{\mathrm{so}}}{m} \mp \frac{\hbar \omega_{0}}{2}\right)^{2}} .
$$

Further, in this limit from (26) we find:

$$
\begin{aligned}
& \tilde{\theta}_{l, k_{x}, \eta=1,4} \rightarrow\left[\begin{array}{c}
\frac{\mathrm{i}}{\Xi}\left[\frac{\hbar^{2} k_{x} k_{\mathrm{so}}}{m}-\frac{\hbar \omega_{0}}{2} \mp \Xi_{1}^{(0)}\left(k_{x}\right)\right] \\
0 \\
0 \\
1
\end{array}\right], \\
& \tilde{\theta}_{l, k_{x}, \eta=2,3} \rightarrow\left[\begin{array}{c}
-\frac{\mathrm{i}}{\Xi}\left[\frac{\hbar^{2} k_{x} k_{\text {so }}}{m}+\frac{\hbar \omega_{0}}{2} \pm \Xi_{2}^{(0)}\left(k_{x}\right)\right] \\
1 \\
0
\end{array}\right] .
\end{aligned}
$$

As a consequence the spinorial Bloch amplitude transforms into a pure spinor without any real space dependence as it can be seen from (15). Expressions (31) and (32) recover the results obtained in Ref. [6].

Finally, let us discuss the polarizations

$$
P_{l, \eta}^{(i)}\left(k_{\mathrm{B}}\right) \equiv\left\langle l, k_{\mathrm{B}}, \eta\left|\hat{\sigma}_{i}\right| l, k_{\mathrm{B}}, \eta\right\rangle,
$$

where $\hat{\sigma}_{i}, i=x, y, z$ are the Pauli spin operators. Writing the identity operator in the $\left\{\left|l, k_{\mathrm{B}}, j, \sigma\right\rangle\right\}$ basis, and taking 
into account the structure of the Bloch spinors (25) and (26) we obtain

$$
\begin{aligned}
& P_{l, \eta}^{(x)}\left(k_{\mathrm{B}}\right)=\sum_{j=0}^{1} \sum_{\sigma^{\prime}, \sigma^{\prime \prime}=-1}^{+1}\left[\theta_{l, k_{\mathrm{B}}, \eta}^{*}\left(j, \sigma^{\prime}\right) \times\right. \\
& \left.\times\left(1-\delta_{\sigma^{\prime}, \sigma^{\prime \prime}}\right) \theta_{l, k_{\mathrm{B}}, \eta}\left(j, \sigma^{\prime \prime}\right)\right]=0, \quad \forall l, k_{\mathrm{B}} \in \mathrm{B} . \mathrm{Z} . \\
& P_{l, \eta}^{(y)}\left(k_{\mathrm{B}}\right)=\sum_{j=0}^{1} \sum_{\sigma^{\prime}, \sigma^{\prime \prime}=-1}^{+1}\left[\theta_{l, k_{\mathrm{B}}, \eta}^{*}\left(j, \sigma^{\prime}\right) \mathrm{i}^{\sigma^{\prime \prime}} \times\right. \\
& \left.\times\left(1-\delta_{\sigma^{\prime}, \sigma^{\prime \prime}}\right) \theta_{l, k_{\mathrm{B}}, \eta}\left(j, \sigma^{\prime \prime}\right)\right]=0, \quad \forall l, k_{\mathrm{B}} \in \mathrm{B} . \mathrm{Z} .,
\end{aligned}
$$

where $\eta=1,2,3,4$. The last two equations show that the longitudinal, that is along the wire, and the perpendicular to the 2DEG plane components of the polarization identically vanish. However, the polarization along the in-plane confinement direction has a finite value:

$$
\begin{aligned}
P_{l, \eta}^{(z)}\left(k_{\mathrm{B}}\right) & =\sum_{j=0}^{1} \sum_{\sigma=-1}^{+1} \theta_{l, k_{\mathrm{B}}, \eta}^{*}(j, \sigma) \sigma \theta_{l, k_{\mathrm{B}}, \eta}(j, \sigma), \\
\forall l, k_{\mathrm{B}} & \in \text { B.Z. }, \eta=1,2,3,4 .
\end{aligned}
$$

From eqs. (37) and (25) we derive the polarizations in the four Bloch sub-bands formed out of the truly 1D Bloch band with index $l$ :

$$
\begin{aligned}
& P_{l, \eta=1,4}^{(z)}\left(k_{\mathrm{B}}\right)=N_{l, k_{\mathrm{B}}, \eta=1,4}^{-1} \times \\
& \times\left\{\frac{1}{\Xi^{2}}\left[\varepsilon_{l}^{-}\left(k_{\mathrm{B}}\right)-\frac{\hbar \omega_{0}}{2} \mp \Xi_{l_{1}}\left(k_{\mathrm{B}}\right)\right]^{2}-1\right\}, \\
P_{l, \eta=2,3}^{(z)}\left(k_{\mathrm{B}}\right)=N_{l, k_{\mathrm{B}}, \eta=2,3}^{-1} \times & \\
\times & \left\{1-\frac{1}{\Xi^{2}}\left[\varepsilon_{l}^{-}\left(k_{\mathrm{B}}\right)+\frac{\hbar \omega_{0}}{2} \pm \Xi_{l_{2}}\left(k_{\mathrm{B}}\right)\right]^{2}\right\} .
\end{aligned}
$$

Using (29) and equalities $\varepsilon_{l}^{-}\left(k_{\mathrm{B}}\right)=-\varepsilon_{l}^{-}\left(-k_{\mathrm{B}}\right)$, $\Xi_{l_{1,2}}\left(k_{\mathrm{B}}\right)=\Xi_{l_{2,1}}\left(-k_{\mathrm{B}}\right)$, the symmetry relation for the polarizations

$$
P_{l, \eta=1,4}^{(z)}\left(k_{\mathrm{B}}\right)=-P_{l, \eta=2,3}^{(z)}\left(-k_{\mathrm{B}}\right),
$$

is derived $\forall l, k_{\mathrm{B}} \in \mathrm{B} . Z$. This symmetry is clearly seen in figs. 3 and 4 , where the four polarizations (38) and (39) are plotted for $l=1$ and $l=2$, respectively. Fig. 3 also shows that the polarizations do not change sign and never approach unity in the first Bloch band. This picture changes for the polarizations in the second Bloch band (see fig. (4). In this band the polarizations change sign. Also there exist nearly fully spin-polarized domains in the first Brillouin zone. As one can see those domains are the ones where the group velocity takes its largest absolute values. The same happens in the limiting case $U(x) \rightarrow 0$ where the group velocity has infinite values for infinite momentum. Indeed, when $U(x) \rightarrow 0$, from (38) one finds for example that $\lim _{k_{x} \rightarrow \pm \infty} P_{\eta=1}^{(z)}\left(k_{x}\right)=\mp 1$ in agreement with Ref. [6]. Thus in the absence of the periodic potential the states can again be characterized by the spin quantum number for large absolute values of the longitudinal momentum.

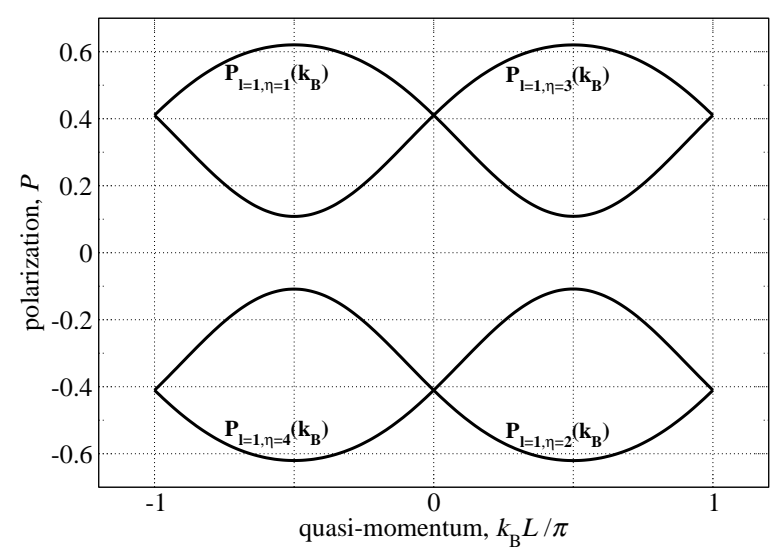

Fig. 3: Spin polarizations along the $z$-axis in the four Bloch sub-bands with $l=1$.

A periodic structure with $V(z)=0$. - In this section we briefly present the resulting energy spectrum when the potential $V(z)$ vanishes and the periodic potential $U(x)$ is arbitrary. Here the solutions of (10) are plane waves, $|j\rangle \equiv\left|k_{z}\right\rangle, \varepsilon_{j}^{z} \equiv \varepsilon_{k_{z}}^{z}=\hbar^{2} k_{z}^{2} / 2 m$ and $\left\langle k_{z}\left|\hat{k}_{z}\right| k_{z}^{\prime}\right\rangle=\delta_{k_{z}, k_{z}^{\prime}} k_{z}$. The diagonalization of eq. (18) leads to the dispersion relations:

$$
\begin{aligned}
& \varepsilon_{l, \eta=1,2}^{2 \mathrm{D}}\left(k_{\mathrm{B}}, k_{z}\right)=\frac{\varepsilon_{l}^{(0)}\left(k_{\mathrm{B}}+k_{\mathrm{So}}\right)+\varepsilon_{l}^{(0)}\left(k_{\mathrm{B}}-k_{\mathrm{so}}\right)}{2}+ \\
& +\frac{\hbar^{2} k_{z}^{2}}{2 m}-\frac{\hbar^{2} k_{\mathrm{so}}^{2}}{2 m} \pm \sqrt{\left(\varepsilon_{l}^{-}\left(k_{\mathrm{B}}\right)\right)^{2}+\left(\frac{\hbar^{2} k_{\mathrm{so}} k_{z}}{m}\right)^{2}}
\end{aligned}
$$

where we have added the upper index $2 \mathrm{D}$ to stress that in this system the energy spectrum is two-dimensional. It can be easily checked that at $k_{z}=0$ eq. (41) gives the same dispersion relation as the one derived from eqs. (20) and (21) in the limiting case $\omega_{0}=0$. For $k_{\mathrm{B}}=0$ and $k_{z}>0$ it follows from (41):

$$
\begin{aligned}
& \varepsilon_{l, \eta=1,2}^{2 \mathrm{D}}\left(k_{\mathrm{B}}=0, k_{z}\right)=\varepsilon_{l}^{(0)}\left(k_{\mathrm{so}}\right)-\frac{\hbar^{2} k_{\mathrm{so}}^{2}}{2 m}+ \\
& +\frac{\hbar^{2} k_{z}^{2}}{2 m} \pm \frac{\hbar^{2} k_{\mathrm{so}} k_{z}}{m} .
\end{aligned}
$$

From eq. (42) one can clearly see that the energy branch with $\eta=2$ has its minimum at $k_{z}=k_{\text {so }}$ for all bands $l$. The splitting of the two branches is linear in $k_{z}$. The last expression also shows that for different band indices $l$ the corresponding energy branches are parallel and there are not anti-crossings. This is also shown in fig. 5 These analytical results are in contrast to what was numerically predicted in Ref. [9].

Finally, in the limiting case $U(x) \rightarrow 0$, we have $\varepsilon_{l}^{(0)}\left(k_{\mathrm{B}}\right) \rightarrow \hbar^{2} k_{x}^{2} / 2 m$ and from eq. (41) it follows:

$$
\varepsilon_{1,2}^{2 \mathrm{D}}\left(k_{x}, k_{z}\right)=\frac{\hbar^{2} k^{2}}{2 m} \pm \frac{\hbar^{2} k_{\mathrm{so}} k}{m},
$$

where $k \equiv|\mathbf{k}|=\sqrt{k_{x}^{2}+k_{z}^{2}}$. One sees that eq. (43) is 


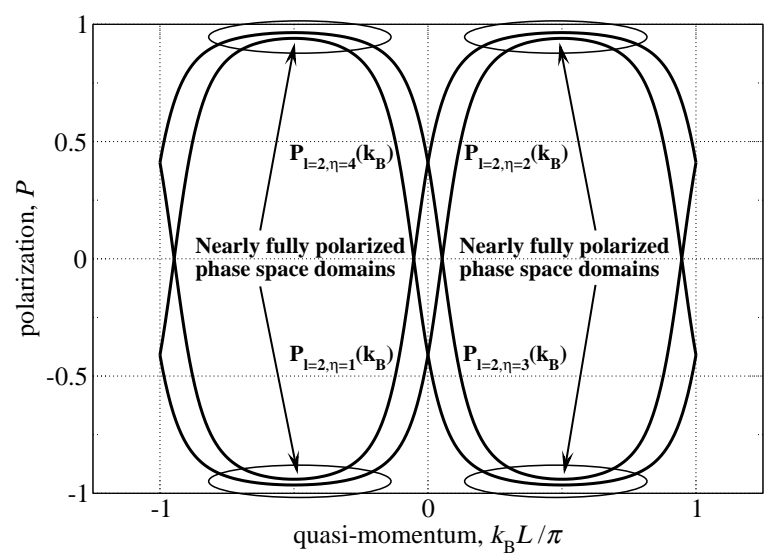

Fig. 4: Spin polarizations along the $z$-axis in the four Bloch sub-bands with $l=2$.

nothing but Rashba's dispersion relation, that is the energy spectrum of Hamiltonian (11) has been recovered.

Materials of interest. - Here we would like to mention that although our theory is general, the concrete results presented on the plots are relevant for III-V compounds. For example in InAs the spin-orbit coupling strength $\alpha \equiv \hbar^{2} k_{\mathrm{so}} / m$ is enhanced up to $4 \cdot 10^{-11} \mathrm{eV} \cdot \mathrm{m}$ as it is demonstrated in Ref. [14]. The effective mass is $m=0.036 m_{0}$. Then for $L$ in the range between $70 \mathrm{~nm}$ and $100 \mathrm{~nm}$ the dimensionless parameter $k_{\mathrm{so}} L=g \pi / 2$ with $g$ being in the range between 0.84 and 1.2.

Conclusion. - We have considered a two-dimensional (2D) electron gas with Rashba's spin-orbit interaction (RSOI) in the presence of two one-dimensional (1D) inplane potentials along mutually orthogonal directions, assuming the first of those potentials to be periodic while making no assumption about the second one. It has been found that in such a system the coordinate part of the Bloch amplitude is the same as the one of the corresponding truly one-dimensional problem without RSOI, however its Bloch's quasi-momentum has a spin-dependent shift proportional to the spin-orbit coupling strength. A general eigen-value problem for the band structure has been presented in terms of the spinorial part of Bloch's amplitude. The cases where the second potential represents either a harmonic confinement or where it vanishes have been studied as applications of the general formalism. For the case of a harmonic confinement with only the first two transverse modes retained analytical relations have been obtained and general symmetry properties of the resulting band structure have been determined. Analytical expressions for the polarizations have been derived as well. Regions of high polarization corresponding to regions of large absolute values of the group velocity have been found. For a vanishing transverse potential exact analytical relations between the energy spectrum of this $2 \mathrm{D}$ system and its truly $1 \mathrm{D}$ problem without RSOI have also been established. We hope that the results of our work

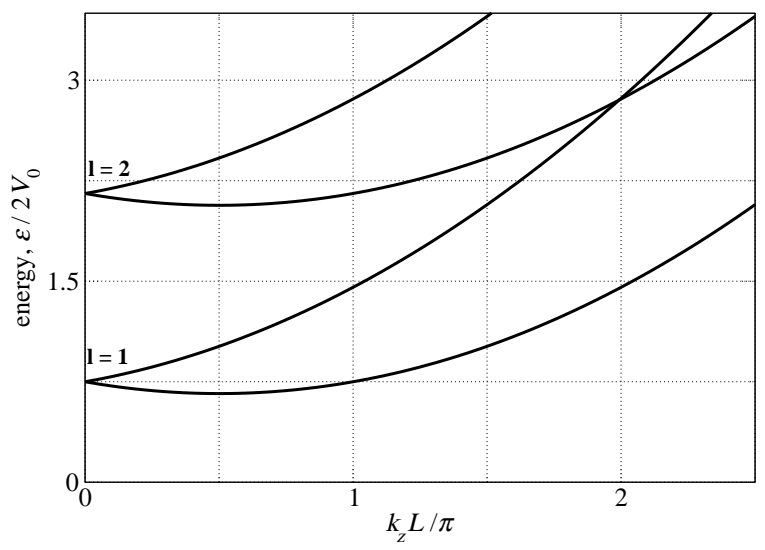

Fig. 5: $k_{\mathrm{B}}=0$ cut of the energy spectrum for a periodic structure with $V(z)=0$. Energy branches with $l=1,2$ are depicted.

could be important to better understand the interplay between RSOI and periodic potentials in a wide range of $2 \mathrm{D}$ and quasi-1D systems which could be used $e . g$. as effective spin rectifiers [15].

$$
* * *
$$

We thank Prof. K. Richter for fruitful discussions. Support from SFB 689 is acknowledged.

\section{REFERENCES}

[1] Rashba E., Fiz. Tverd. Tela (Leningrad), 2 (1960) 1224, Sov. Phys. Solid State, 2 (1960) 1109.

[2] Nitta J., Akazaki T. and Takayanagi H., Phys. Rev. Lett., 78 (1997) 1335.

[3] Dresselhaus G., Phys. Rev., 100 (1955) 580.

[4] Datta S. and Das B., Appl. Phys. Lett., 56 (1990) 665.

[5] Moroz A.V. and Barnes C.H.W., Phys. Rev. B, 60 (1999) 14272; ibid. 61 (2000) R2464.

[6] Governale M. and Zülicke U., Phys. Rev. B, 66 (2002) 073311.

[7] Perroni C.A., Bercioux D., Ramaglia V.M. and Cataudella V., J.Phys.: Condens. Matter, 19 (2007) 186227.

[8] Kleinert P., Bryksin V.V. and Bleibaum O., Phys. Rev. B, 72 (2005) 195311.

[9] Demikhovski V.YA. and Khomitsky D.V., Pis'ma $Z h$. Eksp. Teor. Fiz., 83 (2006) 399.

[10] Demikhovskit V.Ya. and Perov A.A., Europhys. Lett., 76 (2006) 477.

[11] Ashkroft N.W. and Mermin N.D., Solid State Physics (Saunders College, Philadelphia) 1976.

[12] Landau L.D., Lifshitz E.M. and Pitaevski L.P., Course of Theoretical Physics, Statistical Physics. Part 2: Theory of the condensed state, Vol. 9 (ButterworthHeinemann, Oxford) 2002.

[13] Luo J., Munekata H., Fang F.F. and Stiles P.J., Phys. Rev. B, 41 (1990) 7685.

[14] Grundler D., Phys. Rev. Lett., 84 (2000) 6074.

[15] Scheid M., Pfund A., Bercioux D. and Richter K., arXiv: cond-mat/0601118. 